\title{
Die Arbeit mit mehrfachgeschädigten alkoholabhängigen Menschen in einer „nassen“ Einrichtung der Suchtkrankenhilfe
}

Volker Frings-Wikker

\author{
The Work With Manifold Handicaped Alcohol-Addicted People In A „Wet“ \\ Arrangement of the „Suchtkrankenhilfe“
}

\section{Zusammenfassung}

Der Artikel beschreibt die Arbeit einer stationären Einrichtung gem. §39 BSHG mit chronisch suchtmittelabhängigen Menschen, die zu einer abstinenten Lebensführung zumindest bei der Aufnahme in der Einrichtung nicht in der Lage sind. Dieses Angebot richtet sich vorwiegend an suchtkranke Menschen aus dem Bereich der Wohnungslosenhilfe. Das Haus wurde 1999 in Betrieb genommen und im Jahr 2001 um eine Außenwohngruppe erweitert. Es versteht sich als niedrig schwelliges Angebot der Suchtkrankenhilfe. Grundlage dieser Arbeit bildet zunächst die Sicherung des Überlebens und darauf aufbauend eine Reduktion des Konsums und Hinführung zur Abstinenz.

\section{Schliuisselwörter}

Reduktion des Alkoholkonsums · Tagesstruktur · Bindeglied zwischen Suchtkrankenhilfe und Gefährdetenhilfe

\section{Abstract}

The following artile describes the work of an full-time arrangement according to $\S 39$ BSHG for chronical alcohol- or medicament-addicted people, who aren't able to cope with tetotalism for the present. Moreover, this offer preponderant for homeless addicts.

The house started its work in 1999 and in 2001 a "Außenwohngruppe" was created. The basic of this work is at first the securation of survival, constructive on that a reduction of consumption and achievement of tetotalism.

\section{Key words}

Reduction of consumption of alcohol · daily structur · connecting link between „Suchtkrankenhilfe“ and „Gefährdetenhilfe“

\section{Peter-Beier-Haus}

Einrichtung für alkoholabhängige Frauen und Männer

Das Diakoniewerk Duisburg als Träger des Peter-Beier-Hauses wurde im Jahr 1979 gegründet. Die GmbH betreibt mehrere Einrichtungen der Suchtkranken- und Gefährdetenhilfe. Die Einrichtungen der Gefährdetenhilfe im Werk machten über viele Jahre die Erfahrung, dass eine Gruppe von Menschen nach einer Vermittlung in suchttherapeutische Einrichtungen in die Einrichtungen der Gefährdetenhilfe zurückkehrte, da sie dem in den Einrichtungen der Suchtkrankenhilfe vorgegebenen Abstinenzgebot nicht folgen konnte. Ausgehend von diesen Er- fahrungen wurden im Diakoniewerk Duisburg Überlegungen angestellt, wie diesen „Drehtürklienten“ ein angemessenes Hilfeangebot gemacht werden kann. Aus diesen Überlegungen erwuchs die Konzeption des heutigen Peter-Beier-Hauses. Das Stammhaus wurde im Jahr 1999 in Betrieb genommen und im Juli 2001 um eine Außenwohngruppe (AWG) erweitert. Als stationäres Angebot der Eingliederungshilfe werden die Pflegesätze gem. §39/40 BSHG vom überörtlichen Träger der Sozialhilfe finanziert.

Betreut werden im Stammhaus 4 Frauen und 14 Männer. In der stationären AWG leben heute 6 Männer. 
Sowohl im Stammhaus als auch in der AWG stehen den Bewohnern Einzelzimmer und Gemeinschaftsräume zur Verfügung. Das Stammhaus ist darüber hinaus mit einer Werkstatt für handwerkliche Beschäftigungen ausgestattet.

Der Verbleib in der Einrichtung ist zeitlich grundsätzlich nicht befristet und richtet sich nach dem individuellen Hilfebedarf.

Beide Einrichtungsteile befinden sich im Stadtgebiet Duisburg. Die Entscheidung, das Angebot im städtischen Bereich anzusiedeln, wurde bewusst getroffen, da viele Betroffene aus dem städtischen Bereich kommen und nicht bereit sind, die Stadt zu verlassen.

Zusammen mit einer abstinenzorientierten Einrichtung des gleichen Trägers versteht sich die Einrichtung als Verbund, der der betreuten Klientel ein auf die individuelle Situation abgestimmtes Angebot machen will. Zwischen diesen Einrichtungen findet eine enge Zusammenarbeit statt.

\section{Zielgruppe}

Das Hilfeangebot des Peter-Beier-Hauses wendet sich an alkohol- und medikamentenabhängige Menschen, die zum Zeitpunkt der Aufnahme nicht in der Lage sind, suchtmittelfrei zu leben. Viele der Bewohner haben in der Vergangenheit an mehreren medizinischen Rehabilitationsmaßnahmen und soziotherapeutischen Maßnahmen teilgenommen, ohne dass eine langfristige Abstinenz erreicht werden konnte (Tab.1).

Jedoch werden auch Bewohner aufgenommen, die in der Vergangenheit noch keinerlei Bezüge zum Suchthilfesystem aufbauen konnten und eine abstinente Lebensform aus heutiger Sicht für sich (noch) nicht akzeptieren können. Neben der Alkoholerkran-

Tab. 1 Verteilung der Vorbehandlungen (bezogen auf die 2002 betreute Klientel)

\begin{tabular}{lllll}
\hline & $\begin{array}{l}\text { Anzahl der medizinischen } \\
\text { Rehabilitationsmaßnahmen } \\
\text { abgeschlossen }\end{array}$ & $\begin{array}{l}\text { Anzgebrochen soziotherapeutischer } \\
\text { Maßnahmen }\end{array}$ \\
$\begin{array}{l}\text { abgeschlossen } \\
\text { abgebrochen }\end{array}$ \\
\hline $\begin{array}{l}\text { Anzahl der } \\
\text { Maßnahmen }\end{array}$ & 10 & 5 & 1 & 4 \\
$\begin{array}{l}\text { Anzahl der } \\
\text { jeweiligen } \\
\text { Bewohner }\end{array}$ & 6 & 3 & 1 & 4 \\
\hline
\end{tabular}

Tab. 2 Dauer der Wohnungslosigkeit vor Aufnahme in der Einrichtung (bezogen auf die 2002 betreute Klientel)

\begin{tabular}{|c|c|c|}
\hline & Wohnungslosigkeit & Untermietverhältnis \\
\hline 2-3 Jahre & - & 1 Bew. \\
\hline 3-6 Jahre & 4 Bew. & 3 Bew. \\
\hline mehr als 6 Jahre & 12 Bew. & 5 Bew. \\
\hline
\end{tabular}

kung kommt i.d.R. eine langjährige Wohnungslosigkeit (siehe Tab. 2) verbunden mit meist vielfältigen Heimerfahrungen hinzu.

Anhand dieser Ausführungen wird deutlich, dass das Angebot ein Bindeglied darstellt zwischen den Bereichen Gefährdetenhilfe und Suchtkrankenhilfe.

Das Durchschnittsalter der im Jahr 2001 betreuten männlichen Bewohner betrug 60,7 Jahre. Bei den Frauen lag das Durchschnittsalter mit 61,1 Jahren knapp darüber. Die Spannbreite bei diesen Durchschnittsberechnungen liegt zwischen 46 und 69 Jahren. Zehn dieser Bewohner ist ein gesetzlicher Betreuer zur Seite gestellt. 58,3\% der im Jahr 2002 betreuten Bewohner weisen eine Vorbehandlung auf (vgl. Tab.1).

Durch den jahrelangen exzessiven Alkoholmissbrauch weisen die Bewohner teilweise unterschiedliche neurologische und organische Schädigungen auf (z.B. Polyneuropathien, Magenerkrankungen etc.). Meist verfügen sie bei der Aufnahme über keinerlei Kontakte zur Familie oder früheren Bekannten und wurden in der Vergangenheit auch im Kreis der Wohnungslosen aufgrund der starken Verhaltensauffälligkeiten ausgegrenzt. Zudem sind die betreuten Menschen in der Regel bereits seit vielen Jahren aus Arbeitsprozessen ausgegliedert (siehe Tab. 3).

Ein Großteil ist kaum in der Lage, den alltäglichen Anforderungen wie Strukturierung von Zeit, Hygiene, Selbstversorgung selbständig zu genügen.

Ausschlusskriterien für die Aufnahme in der Einrichtung ist das Vorliegen einer Pflegebedürftigkeit im Sinne der Pflegeversicherung über Pflegestufe 1 hinaus. Weitere Ausschlusskriterien bilden eine akute psychotische Erkrankung sowie Suchterkrankungen aus dem Bereich illegaler Drogen.

\section{Zielsetzungen}

Grundsätzliches Ziel der Arbeit ist die Heranführung an die Alkoholabstinenz. Die oben beschriebene Lebenssituation der betreuten Klientel lässt jedoch erahnen, dass diese Zielsetzung zumindest zum Zeitpunkt der Aufnahme nicht für alle Bewohner erreichbar erscheint. Bei der Aufnahme wird den Bewohnern deshalb auch nicht ein Bekenntnis zur Abstinenz abverlangt. Im Mittelpunkt der Arbeit steht zunächst die Reduktion des Konsums (harm reduction). Hiervon ausgehend können die Zielsetzungen wie folgt zusammengefasst werden:

- die Absicherung der Grundbedürfnisse

- Linderung körperlicher Folgeschäden

- Einsicht in die Grunderkrankung

Tab. 3 Dauer der Arbeitslosigkeit (bezogen auf die 2002 betreute Klientel)

\begin{tabular}{|lc|}
\hline letzte Berufstätigkeit & Anzahl der Bewohner \\
\hline bis zu 10 Jahre & 8 \\
\hline mehr als 10 Jahre & 16 \\
\hline
\end{tabular}


- Veränderung des Konsumverhaltens

- Reduktion der Trinkmenge

- Reduktion von Trinkexzessen

- Hinführung zur Abstinenz

\section{Die Arbeit innerhalb der Einrichtung}

Der Aufbau einer stabilen Beziehung stellt in der Arbeit eine wichtige Voraussetzung für eine tragfähige Zusammenarbeit dar. Unsere Arbeit mit den Bewohnern folgt dem Grundsatz „Wollen setzt Können voraus“. Konkret heißt dies, dass die Zielsetzungen bei den vorhandenen Fähigkeiten des Bewohners ansetzen und in einem gemeinsamen Hilfeplan festgeschrieben werden, der regelmäßig überprüft und ggf. verändert wird. Inhalte dieses Hilfeplanes sind Zielsetzungen (z.B. Reduktion des Konsums, Kontaktaufbau zu Verwandten) und Schritte zur Umsetzung, die mit dem Bewohner erarbeitet werden.

In der Regel gilt es, beim Bewohner zunächst ein Problembewusstsein für seine Sucht zu entwickeln und Zusammenhänge zwischen der Lebenssituation und der Suchtproblematik aufzudecken und somit eine Veränderungsmotivation zu wecken. In diesem Prozess wird dem Bewohner vermittelt, dass er der beste Experte für sich ist und Entscheidungen durch ihn selber getroffen werden müssen. Dies geschieht vor der Überzeugung, dass der Bewohner nicht vollständig unfähig ist, sich zu steuern. Vielmehr gilt es, die noch vorhandenen Fähigkeiten zur Eigensteuerung und Selbstkontrollmöglichkeiten zu entdecken und zu unterstützen.

Die Ausweitung alkoholfreier/alkoholreduzierter Intervalle und damit verbunden die Gewinnung positiver Erfahrungen sind eine wichtige Weichenstellung für die Arbeit mit dem Bewohner. Neben der Regel, dass während der strukturierten Aktivitäten im Tagesverlauf nicht konsumiert wird, ist die Reduktion beim einzelnen Bewohner auf individuelle Vereinbarungen zurückzuführen. Diese Vereinbarungen reichen von festgelegten Trinkmengen zu bestimmten Tageszeiten, die vom Bewohner selbständig überwacht werden, bis hin zu Trinkmengen, die durch Mitarbeiter überwacht werden müssen. Die selbständige Einteilung erfolgt hierbei teilweise durch das Führen eines eigenen Trinktagebuches. Jedoch stellt die eigene Kontrolle für einige Bewohner eine Anforderung dar, zu der sie derzeit nicht in der Lage sind und weshalb sie Unterstützung von Seiten der Mitarbeiter erhalten.

Ein wesentliches Element zur Ausgestaltung dieser Arbeit bildet die Tagesstruktur innerhalb der Einrichtung. Diese Struktur ist gekennzeichnet durch verpflichtende Angebote (z.B. regelmäßige Gespräche, Mitarbeit im Haus, Gruppenangebote) für die Bewohner und freiwillige Angebote (z.B. Spielenachmittage, Ausflüge), die je nach Interessenlage genutzt werden können. Die Gleichmäßigkeit der Tagesstruktur dient dazu, den Ablauf für unsere Bewohner überschaubar zu halten. Somit gibt er insbesondere Bewohnern mit hirnorganischen Veränderungen Sicherheit und Orientierung. Im Rahmen dieses Tagesablaufes können verloren gegangene Fähigkeiten (z.B. Umgang mit Geld, sorgsamer Umgang mit dem eigenen Körper) wieder erlernt werden und neue Erfahrungen in der Gemeinschaft gesammelt werden.

Gleichzeitig achten wir darauf, dass der Charakter einer stationären Einrichtung und damit verbunden der Grad der Versorgung möglichst gering gehalten wird. Für die konkrete Arbeit bedeutet dies, dass Bewohner entsprechend ihrer Fähigkeiten an eine Eigenversorgung herangeführt werden.

\section{Ausblick}

Aufgrund der schweren Schädigungen der betreuten Klientel kann nicht erwartet werden, dass eine dauerhafte Abstinenz in dem relativ kurzen Zeitraum des Bestehens der Einrichtung bei einem Großteil der Bewohner zu erreichen ist. Drei Bewohner leben inzwischen innerhalb der Einrichtung abstinent und haben für sich diese Lebensform als die gesündeste anerkannt. Diese Abstinenzphasen reichen zwischen 8 und 28 Monaten.

Neben der Reduzierung der Trinkmengen ist jedoch die Bereitschaft des Bewohners, sich in die Gemeinschaft einzubringen, wesentlicher Maßstab für die Arbeit. Wir gehen davon aus, dass das zufriedene Leben in der Gemeinschaft auch ein wesentlicher Faktor für den Rückgang des Alkoholkonsums ist.

Es wäre verfehlt zu behaupten, dass unsere Klientel grundsätzlich einem standardisierten Programm zum kontrollierten Trinken eigenständig folgen könnte, da unsere Erfahrungen zeigen, dass viele Bewohner einer äußeren Kontrolle bedürfen. Diesen Anspruch können und dürfen wir aufgrund der vielfältigen Schädigungen der Bewohner nicht haben. Mit diesem Bericht soll auch nicht in Abrede gestellt werden, dass Abstinenz die gesündeste Lebensform für die von uns betreuten Menschen ist. Jedoch vor dem Hintergrund, dass dieses Ziel zur Zeit nicht bei allen Bewohnern umsetzbar ist, erscheint es sinnvoll, einen niedrig schwelligen Zugang zum Hilfesystem anzubieten und somit eine Form der harm reduction in die Arbeit mit mehrfachgeschädigten alkoholabhängigen Menschen einzuführen. 